\title{
Huellas de descarnado en el Paleolítico Superior: la cueva de El Castillo (Cantabria)
}

\author{
P. Pumarejo y V. Cabrera *
}

El estudio de las marcas encontradas en los huesos, fundamentalmente en forma de cortes o incisiones provee un importante campo de contraste de las técnicas de descuartizamiento y carnicería de un animal. Este tipo de estudios, ha sido utilizado desde hace mucho tiempo como prueba de las actividades antropicas sobre restos óseos (LARTET 1860; DART 1957; ClaRK 1972). Sin embargo es el trabajo de Binford (1981) el que plantea la necesidad de un estudio sistemático de las marcas y cortes sobre los huesos. Hasta hace poco tiempo este tema ha sido uno de los menos tratados por los investigadores, si bien cabe destacar los estudios realizados también por Albrecht, Berke y Poplin (1983), Lyman (1987) y, los recientemente realizados en nuestro pais por Reixach (1986).

El material óseo que presentamos en este artículo forma parte de la Tesis Doctoral de uno de nosotros (P.P.) basada en tres yacimientos paleolíticos de Cantabria: El Castillo (Puente Viesgo), El Juyo (Igollo) y La Pila (Cuchia). La elección de estos yacimientos está justificada por varios motivos. En primer lugar, se tratan todos ellos de yacimientos en cueva excavados recientemente o en curso de excavación, por lo que ha sido sistemática la recogida de sus materiales. Por otro lado las nuevas técnicas de recogida de restos vegetales aplicadas en estos yacimientos testifican la importancia que las actividades cinegéticas tuvieron para sus ocupantes. Al mismo tiempo, se intenta trabajar con una secuencia amplia del Paleolitico Superior Cantábrico en la que poder observar, si las hubiera, diferencias temporales entre sus complejos culturales.

\footnotetext{
* Dpto. de Prehistoria e Historia Antigua, U.N.E.D
} 


\section{HIPÓTESIS DE PARTIDA}

En sintesis la finalidad de todo el trabajo parte de una serie de premisas, si bien esta comunicación se basa en la constatación de las dos primeras:

La primera hipótesis contempla la posibilidad de la existencia de diferentes tipos de incisiones y de distintas localizaciones anatómicas de las mismas, dependiendo del trabajo realizado sobre el animal desde su abatimiento hasta su consumo, entendiendo como cadena técnica de aprovechamiento la sucesión de las siguientes actividades: separación de la piel $\rightarrow$ despiece $\rightarrow$ desarticulación $\rightarrow$ descarnado $\rightarrow$ fracturación.

En segundo lugar se establece que la posición anatómica de las incisiones, si han sido provocadas por el aprovechamiento económico de la pieza, ha de ser coherente con las actividades realizadas. Ello las diferenciará de las marcas producidas por animales, raices, etc. Así aparecerán, principalmente, en las zonas de inserción de tendones y ligamentos, cerca de las epifisis óseas, lugares donde hay que incidir para despiezar y desarticular los miembros.

Como tercera hipótesis se trata de ver la posible relación, por un lado, entre la edad de los animales cazados y, por otro, las partes anatómicas de animal que aparecen en el yacimiento, y la presencia o no de incisiones sobre los huesos.

En cuarto lugar, y como ampliación a la hipótesis anterior, se plantea la posible relación entre la localización geográfica del yacimiento y el transporte de la caza del mismo.

Como quinta hipótesis se plantea la posibilidad de encontrar diferentes modelos de despiece desde un punto de vista sincrónico, según la especie cazada y el área geográfica, y desde una perspectiva diacrónica, según el complejo cultural que se examine.

En último lugar se tiene en cuenta la posible existencia de una distribución espacial de los restos con incisiones sobre la superficie de los niveles de ocupación, como resultado de la distribución del espacio de habitación según las actividades realizadas en él.

\section{UN CASO DE ESTUDIO: LA CUEVA DEL CASTILLO (PUENTE VIESGO)}

El presente trabajo está realizado sobre un muestreo aleatorio entre los restos óseos del nivel basal del auriñaciense de la cueva del Castillo 
(Puente Viesgo), en curso de excavación y de estudio. Este análisis va encaminado a fijar los criterios del modelo observado en los niveles del Magdaleniense Superior y Azilienses de la cueva de la Pila. Con ello se intenta observar si existe un cambio de comportamiento entre dos momentos culturales diferentes, uno del inicio y otro del final que pueden encuadrar la evolución del hábito, si lo hubiere, a lo largo del Paleolítico Superior.

Después de haber analizado un gran número de restos óseos de la cueva de La Pila (Cuchia, Cantabria), cuya estratigrafia permite conocer en detalle la transición Magdaleniense/Aziliense del Cantábrico (BERNALDO DE Quiros et alii 1988), los resultados que hemos obtenido, en lo que a morfologia y situación anatómica de las incisiones se refiere, son similares a los de los estudios de los que hemos partido.

Hemos sistematizado las incisiones en varios "modelos" señalando las caracteristicas de las incisiones y su relación con la situación anatómica según el tipo de hueso de que se trate, sin diferenciar niveles arqueológicos ni especies animales, con el fin de poder tener unos primeros resultados para poder enfocar nuestra tarea de investigación ( $\mathrm{Pu}$ MAREJO Y BERNALDO DE QUIRÓS 1990).

Con el fin de comprobar si los modelos obtenidos se repiten en otros yacimientos de caracteristicas diferentes al que estamos estudiando, y moviéndonos, principalmente, en el campo de las dos primeras hipótesis, hemos intentado aplicar el análisis a una muestra amplia de los restos paleontológicos de la cueva de El Castillo (Puente Viesgo, Cantabria) logrando resultados positivos.

Castillo, representa un enclave excepcional como cazadero. Domina el ancho valle del Pas formando un cerrojo con el monte frontal que pertenece al mismo sistema del Dobra. Al mismo tiempo desde el yacimiento a uno $130 \mathrm{mts}$ sobre el rio puede rodearse el monte alcanzando dos valles transversales más angostos, con cursos fluviales, que remontandolos se llega al valle del Besaya.

Siguiendo el curso del Pas se alcanza la costa, a unos $25 \mathrm{~km}$. En la desembocadura el biotopo es diferente, formándose ensenadas y medios de dunas frente al sistema kárstico donde se ubica la cueva de la Pila.

La comparación del modelo, por lo tanto es importante ya que entrecruza no sólo dos momentos culturales diferentes, ambos de transición, sino también dos yacimientos con entornos muy diferentes aunque muy próximos y pertenecientes a la misma cuenca fluvial.

Los restos óseos de Castillo estudiados pertenecen a la unidad estratigráfica (18b2, 18c y 19 sup.) que se está excavando en la actualidad y 
para la cual se han obtenido dataciones por el acelerador de partículas que rodean los 40.000 años (CABRERA y Bischoff 1989).

La cantidad de la muestra analizada es inferior a la procedente de la cueva de la Pila, como se muestra en la tabla siguiente:

\begin{tabular}{lcccc}
\hline & \multicolumn{1}{c}{ TFE } & RFA & TRI \\
\cline { 2 - 3 } La Pila & $(+) 9.000$ & 4.800 & 1.500 \\
Castillo & $(+) 20.000$ & 2.000 & 400 \\
\hline
\end{tabular}

(TFE: Total Estimado de restos de Fauna; RFA: Restos de Fauna Analizados; TRI: Total Restos con Incisiones).

Existe diferencia en la proporción entre ambos yacimientos entre las incisiones halladas y los restos de fauna analizados (La Pila: $31,25 \%$. Castillo: $20 \%$ ). El porcentaje es muy alto en ambos, para la muestra analizada hasta el momento.

En Castillo hemos encontrado los determinados tipos de incisiones distribuidos en las siguientes partes del esqueleto procedente en su mayoria de restos de Cervus elaphus.

\section{Cráneo}

Los cráneos muestran incisiones cortas y paralelas en la superficie de los temporales, derivadas de la separación de la piel, y en el diastema del maxilar.

\section{Mandibulas y maxilares}

Las mandibulas y los maxilares pueden presentar incisiones largas transversales y oblicuas en la zona alveolar, tanto en su cara interna como en la externa, en grupos generalmente de dos a cinco cortes aunque no falten los ejemplos con mayor frecuencia (fig. 1.1 - 1.2). Es probable que la marcas de la cara interna de las mandibulas estén relacionadas con la extracción de la lengua del animal y que las de la externa se hayan producido al cortar el músculo bucinador. 


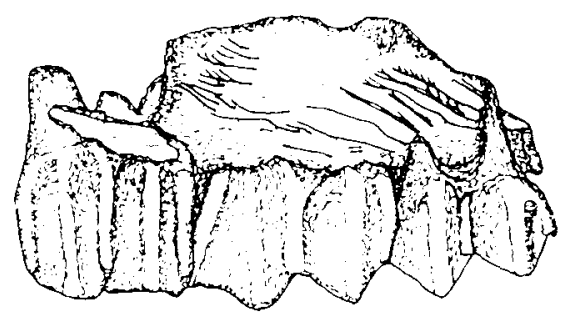

$1 \cdot 1$

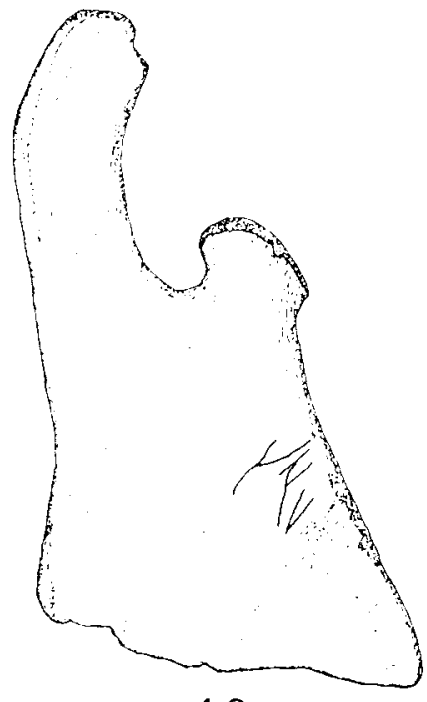

$1 \cdot 3$

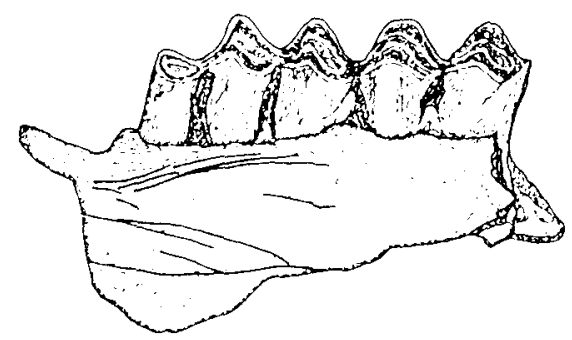

$1 \cdot 2$
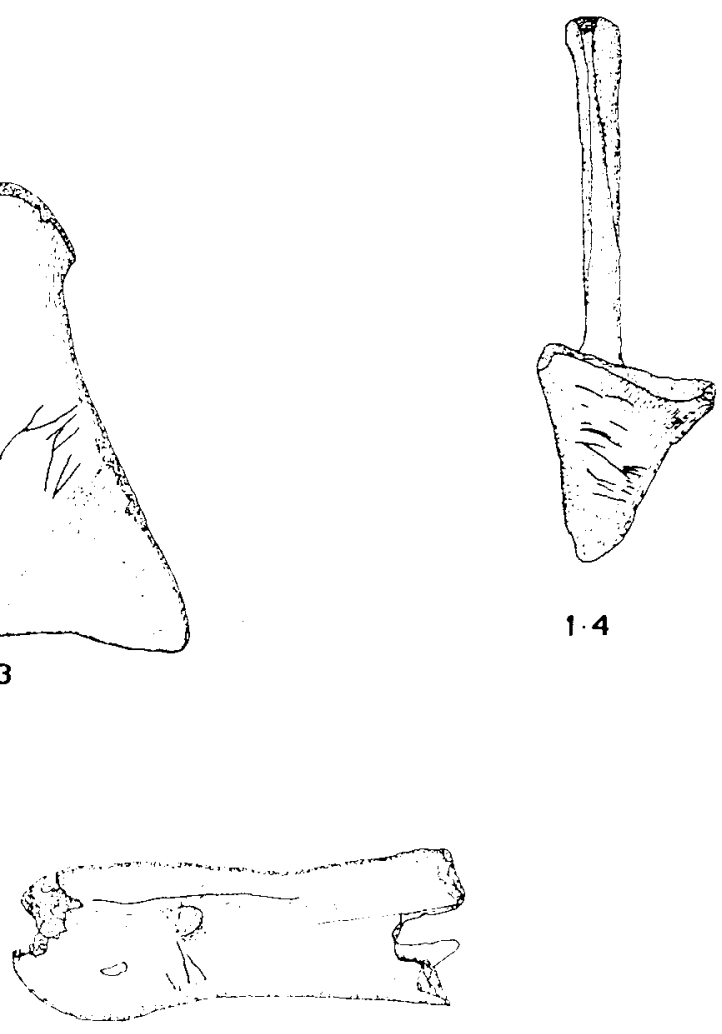

1.5

Fig. 1. Restos craneales. 1.1. maxilar: incisiones en la cara externa, zona alveolar. 1.2. mandibula: incisiones en la cara interna, zona alveolar. 1.3. rama ascendente: incisiones cerca del cóndilo mandibular. 1.4. forámen mentoniano. 
En las ramas ascendentes se observan incisiones en el cóndilo mandibular o muy cerca de él, generalmente en la cara externa, de delineación transversal u oblicua, de pequeño tamaño y paralelas entre si (Fig. 1.3). Son cortes realizados para seccionar el músculo masetero —uno de los músculos básicos para el cierre de la mandíbula- y los ligamentos lateral y caudal de la articulación mandibular que unen la mandibula con el arco cigomático. Estos cortes permiten separar la mandibula inferior de la fosa temporal y por lo tanto del resto de la cabeza.

También pueden encontrarse incisiones cortas o largas, transversales y oblicuas alrededor del foramen del mentón como consecuencia de los cortes dados para separar del hueso la región mentoniana (fig. 1.4).

\section{Vértebras torácicas}

En la base de las apófisis espinosas de las vértebras torácicas suelen aparecer inicisiones indistintamente en una $u$ otra cara e incluso en las dos (Fig. 2.1.) De orientación oblicua y de tamaño irregular, se encuentran en grupos de dos a cinco cortes paralelos entre si, aunque hay algunos grupos más numerosos. Su situación nos hace pensar en que se han producido al realizar cortes para extraer el lomo.

\section{Costillas}

Muestran incisiones transversales $u$ oblicuas paralelas en ambas caras 0 en una de ellas en la zona mesial (fig. 2.2). Generalmente son de pequeño tamaño y se asocian en grupos de dos a cinco o a veces mayores. Las incisiones de la cara externa se originan, dependiendo del lugar que ocupe la costilla, bien por los cortes que han de realizarse para desconexionar el miembro anterior del tronco cortando el músculo serrato o tal vez el músculo extensor grueso del antebrazo (triceps), o bien en el descarnado (fig. 2.3).

En algunas costillas se aprecian incisiones en la cabeza articular con la finalidad de desarticular estos huesos de la columna vertebral.

\section{Omóplatos}

En estos huesos se observan incisiones transversales, cortas y paralelas, en el borde inferior del cóndilo y en el cuello de la escápula. A veces tienen marcas longitudinales, transversales u oblicuas, de tamaño variable, paralelas o subparalelas, a lo largo de la base de la espina, en la cara externa, tanto en la fosa supraspinosa como en la infraspinosa (fig. 2.4). 


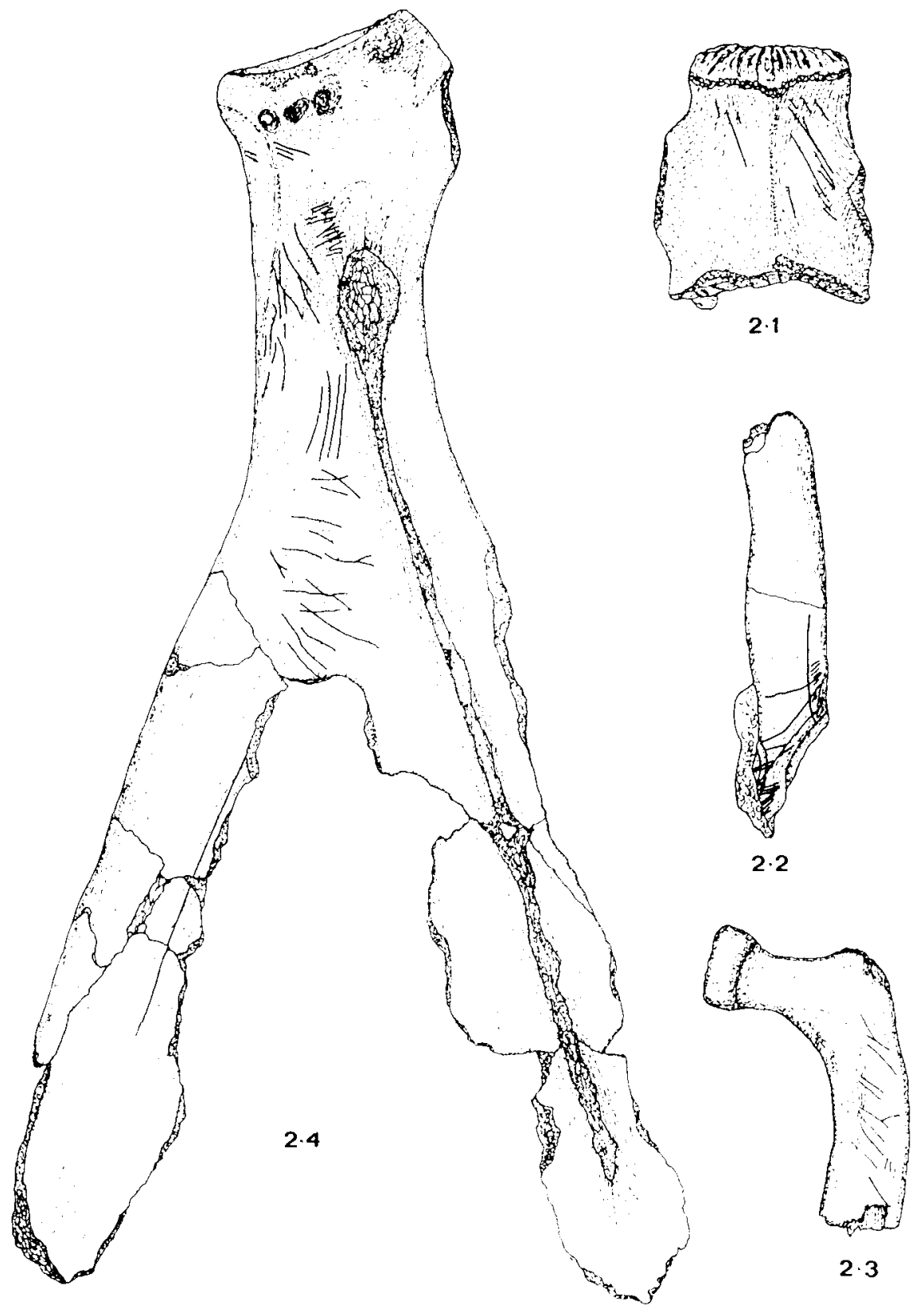

Fig. 2. Restros postcraneales. 2.1. vértebra. 2.2. apófisis espinosa. 2.3. costilla. 2.4. omóplato. 
Las incisiones del cuello de la escápula se localizan en la zona de inserción de biceps, que ha sido cortado para poder separar del tronco el miembro anterior. Las marcas de las fosas supra e infraspinosa, de morfologia diferente, nos indican la realización de un trabajo de descarnado en el omóplato.

\section{Húmeros}

Hasta el momento sólo hemos identificado incisiones en las epífisis distales de los húmeros. Se trata de marcas transversales, cortas y paralelas, en grupos de dos a cinco cortes, que se sitúan en los cóndilos laterales, en las caras externa e interna (fig. 3.2).

Estos cortes se originan al desarticular el miembro anterior separando el húmero del radio, cortando por la cara externa el ligamento colateral del codo y por la interna el ligamento colateral medial del codo.

\section{Cúbitos}

Incisiones oblicuas en la zona proximal, en una o en las dos caras, en la superficie del oleacráneo y cerca de la muesca semilunar, en grupos de dos a cinco, siendo paralelas entre sí.

Las marcas de la superficie del oleacráneo de la cara externa se deben a la separación de este hueso del húmero cortando los músculos corto y mediano del antebrazo. Y los cortes de la muesca semilunar en la cara interna y externa se efectúan al separar la ulna del radio incidiendo sobre el ligamento radiocubital transverso medial y transverso lateral respectivamente.

\section{Tarsales}

De los huesos tarsales por ahora sólo tenemos identificadas incisiones en el escafocuboides, situadas en las caras interna. Son transversales, cortas y paralelas entre si, en grupos de dos a cinco marcas. Su origen está en la separación de los huesos tarsales al incidir sobre los iigamentos largo interno y externo.

\section{Metacarpos y metatarsos}

En ambos huesos se encuentran los mismos tipos de incisiones (figs. 3.3 y 3.4 ). En las epifisis proximales hay marcas transversales cortas y 

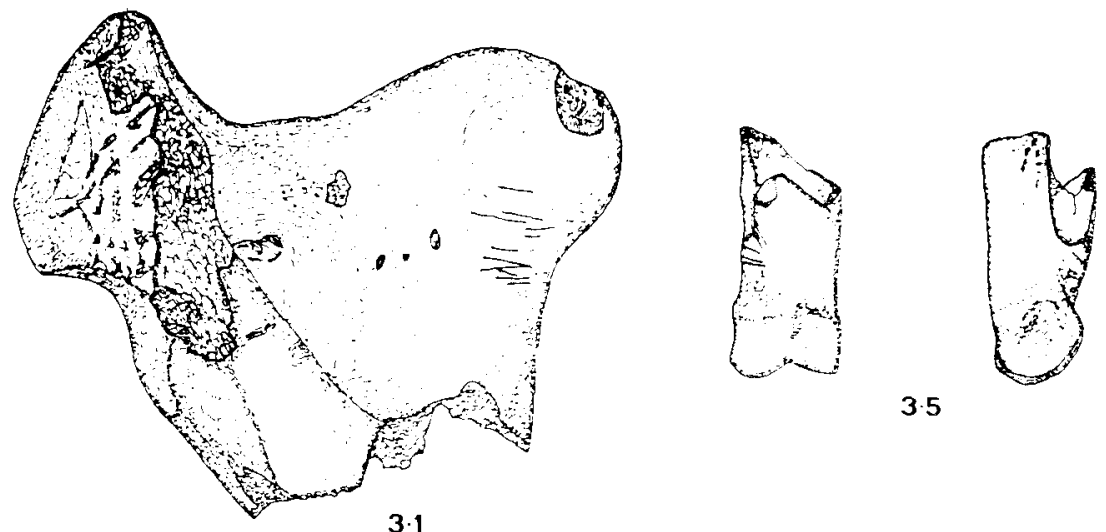

3.5

$3 \cdot 1$
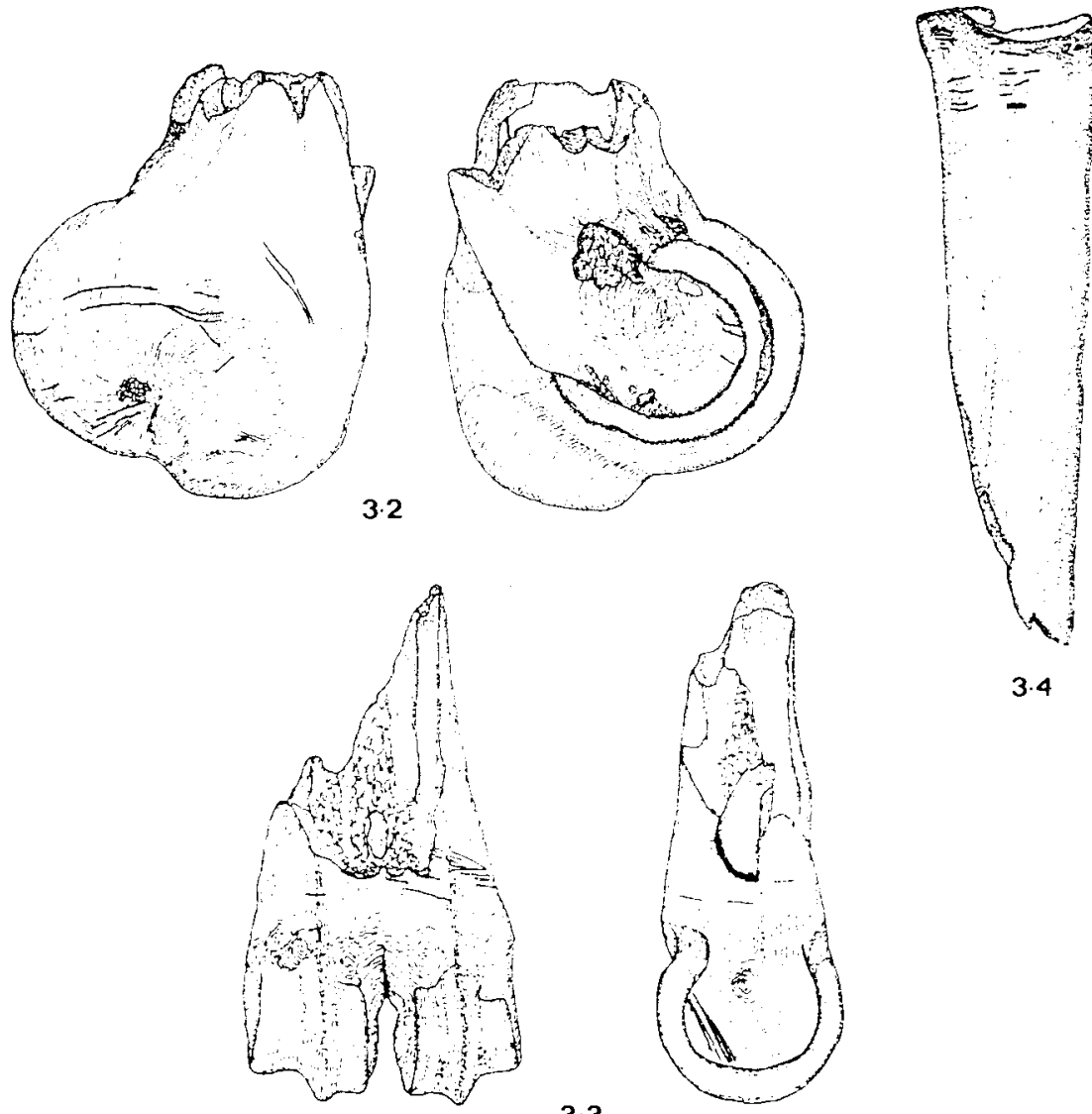

$3 \cdot 3$

Fig. 3. Restos postcraneales. 3.1. fémur. 3.2. húmero. 3.3. metacarpo. 3.4. metatarso. 3.5. primera falange. 
paralelas, en la cara anterior y en los laterales, que se han realizado al cortar en la pata delantera los ligamentos colateral lateral y colateral medial de la articulación intercarpiana, que unen el metacarpo con el radio, y en la pata trasera los ligamentos largo interno y largo externo, que unen el metatarso y la tibia.

En el extremo distal encontramos incisiones en las cuatro caras, todas ellas cortas, aunque puede haberlas largas, transversales y paralelas. Las de las caras interna y externa de ambos huesos se sitúan en el lugar donde se insertan el ligamento sesamoideo colateral lateral y el ligamento colateral lateral del menudillo, que unen el metacarpo y el metatarso con la primera falange de cada extremidad. Las marcas de la cara anterior se han originado al cortar el tendón del músculo extensor anterior de las falanges, y las de la cara posterior al seccionar el músculo flexor superficial.

\section{Primeras falanges}

Marcas transversales, cortas y paralelas en la zona proximal de la cara interna y externa, derivadas de los cortes dados a los ligamentos que unen la primera falange con el metacarpo y metatarso. A veces también se encuentran en la cara posterior por haberse cortado los ligamentos distales o sesamoideos inferiores (fig. 3.5).

En la epífisis distal puede haber incisiones transversales, cortas y paralelas en la cara interna o externa, de haber cortado el ligamento cuartilla-cartilago alar para separar esta falange de la siguiente.

Es probable que algunas de las incisiones que aparecen en las primeras falanges se hayan producido al separar la piel pues en estas zonas se encuentra en contacto directo con el hueso.

\section{Diáfisis}

Por tratarse de fragmentos óseos no identificables resulta dificil precisar la situación de las incisiones, pero teniendo en cuenta la delineación de las fibras que los forman se observa que la gran mayoria de ellas son oblicuas o longitudinales, siendo escasas las transversales. Su longitud es mayor que la del resto de las incisiones y generalmente son paralelas o subparalelas. Estas marcas se derivan del trabajo de descarnado de los huesos largos. 


\section{CONCLUSIONES}

Al comparar los tipos de incisiones observados hasta el momento en las cuevas de El Castillo y de La Pila, se aprecia que en primero de estos yacimientos faltan algunos de los tipos de incisiones sistematizados en el segundo (Pumarejo y Bernaldo de Quirós 1990). Asi en los niveles auriñacienses de $\mathrm{El}$ Castillo por el momento no se han visto incisiones en vértebras cervicales, pélvis, fémures, tibias, calcáneos, astrágalos ni gran cuneiforme.

Esta diferencia podria ser producto de la distancia cronológica que separa estos dos yacimientos y el resultado de un distinto aprovechamiento de la caza en los momentos iniciales del Paleolitico Superior Cantábrico (El Castillo) y en los finales (La Pila). En principio puede explicarse por la reducida cantidad de restos paleontológicos analizados hasta el momento en El Castillo, por tratarse de un primer muestreo que nos ayude a enfocar nuestro posterior trabajo de investigación. Sin embargo, tenemos que valorar otros factores que pueden influir en la carencia de determinados tipos de incisiones.

\section{A) Causas Tafonómicas y conservación diferencial:}

En primer lugar la observación de las incisiones depende del estado de conservación del hueso, pudiendo habar zonas o niveles del yacimiento en los que éste se conserve peor que en otras.

Por otro lado influye la propia conservación diferencial de los restos óseos, que depende directamente de la parte del esqueleto de la que se trate. Ello puede darnos una visión sesgada de la realidad, al no mostrarse algunos de los tipos de incisiones típicos por la destrucción del material se produce el riesgo de interpretar su falta por la no realización en el yacimiento de alguna de las tareas propias del aprovechamiento animal (separación de la piel, despiece, desarticulación, descarnado).

Algunos huesos por su dureza y tamaño, soportan mejor que otros los procesos que pudieran destruirlos durante su manipulación (desarticulación, despiece, etc.) tales como falanges, articulaciones y epifisis de huesos largos, frente a otros de mayor tamaño y fragilidad como cráneos, mandibulas, omóplatos y pelvis, que pueden romperse fácilmente. Además estos dos grupos de huesos también presenta, lógicamente, mayor o menor facilidad a deteriorarse con procesos de incidencia posterior a la deposición, como el pisoteado, la compactación de los sedimentos, etc.

Además, hay que tener en cuenta la posible influencia de factores fi- 
sico-quimicos y biológicos que hayan actuado después de la deposición del registro arqueológico (calcificación, carnivoros, raices).

\section{B) Causas antrópicas}

Otro factor que puede aclararnos este tema es la posible existencia de una selección de las partes anatómicas de los animales aportadas al yacimiento en función, por un lado, de su valor alimenticio, y por otro, de la facilidad de transporte de las piezas abatidas según su tamaño y distancia a la que hayan sido cazadas.

También hay que valorar la composición de la materia prima lo que puede hacer que las incisiones estén más marcadas o no. En este sentido se ha detectado que en el nivel 18b2 las huellas observadas carecen de microsurco, y asimismo la huella es suave. En este nivel la abundancia de piezas liticas asociadas en caliza negra es apreciable por lo que pudiera deberse a este factor, dada la menor dureza de esta materia prima.

En el nivel $18 \mathrm{c}$, en donde la caliza negra alóctona también es abundante, se reproduce el mismo hecho. Sin embargo en el nivel 19 superior, donde apenas si se detecta caliza entre la industria lítica incluidos los debris de dos $\mathrm{mm}$, las huellas están muy marcadas. La correlación por tanto es muy neta, por lo que en estudio de la asociación espacial de la industria litica y los restos de fauna adquiere mayor relieve en este campo. Este estudio nos podrá determinar si se debe a un factor aleatorio, dependiendo del momento y la materia al alcance. O bien si se debe a una distribución debida a distintas actividades dentro de la ocupación.

También hay que contar con la posible diferencia de la fuerza impresa con la que se ha realizado el gesto manual.

Aunque pueda ser prematuro hacer conclusiones se pueden destacar algunas ideas que de momento afianzan nuestras hipótesis de partida. En primer lugar, parece que nuestras hipotesis sobre la existencia de diferentes tipos de incisiones y de distintas localizaciones anatómicas dependiendo del trabajo realizado sobre el animal, y la coherencia de su posición anatómica en función de los puntos de inserción de tendones y ligamentos, se va confirmando a medida que analizamos un mayor número de restos óseos.

La tarea de separación de la piel ha dejado huellas en aquellas regiones anatómicas donde la piel se encuentra más en contacto con los huesos como en el caso de los cráneos y primeras falanges.

El despiece ha dejado marcas en los omóplatos por el desmembramiento de las extremidades anteriores. 
Las labores de desarticulación, que cortan tendones y ligamentos, han incidido sobre las zonas cercanas de las epifisis del húmero, del cúbito, del metacarpo y metatarso, en las mandibulas, en el cuello del omóplato $y$ en las articulaciones de las costillas.

Por último, el descarnado se aprecia en las diáfisis de los huesos largos, en los cuerpos de las costillas, en las fosas supra e infraspinosa de los omóplatos y en las apófisis espinosas de las vértebras.

No parece interesante el hecho de que se repitan en los niveles auriñacienses de la cueva de El Castillo la práctica totalidad de los «modelos" de incisiones definidos en los niveles magdalenienses y azilienses de La Pila, en dos yacimientos de áreas geográficas y de complejos culturales diferentes, lo que nos indica en principio la posibilidad de que no haya durante el Paleolitico Superior Cantábrico una diferenciación ni geográfica ni temporal en las labores de carniceria, manteniéndose una pauta común prácticamente hasta la actualidad.

En el futuro, el análisis se irá completando con los restos procedentes del Paleolitico Medio e Inferior de la gran secuencia de la cueva del Castillo que alcanza cronologias rissienses (CABRERA 1984). De esta manera es posible que en el siglo $x \times 1$ conozcamos mejor los distintos estadios de la cadena técnica de aprovechamiento de los restos de fauna a lo largo de un espectro muy amplio de tiempo, en una región determinada.

\section{BIBLIOGRAFIA}

Albrecht, G., Berke, H. y POPLIN, F. (1983): Recherches scientifiques sur les inventaires Magdaléniens du Peterstels, fouilles 1974-1976. Verlag Archaeologica Venatoria. Universität Tübingen

Bernaldo de Quiros, F. (1980): Notas sobre la economia del Paleolitico Superior. Centro de Investigación y Museo de Altamira. Monografia 1. Santander.

Bernaldo de Quiros, F., Gutiérrez, C. Heras, C., Laguerra, M. A.. Pelayo, M., Pumarejo, P. y UzouIANo, P. (1988): Nouvel!es données sur la transition Magdalénien supérieur - Azilien: la cueva de "La Pila» (Cantabria, Espagne)". Le peuplement magdalénien. Paleographie physique et humaine. Centenaire de la découverte de l'Homme de Chancelade.

BINFORD, L. R. (1981): Bones. Ancient men and modern myths. Studies in Archaeology. New York.

Cabrera, V. (1984): El yacimiento de la cueva de El Castillo (Puente Viesgo. Santander). Bibliotheca Praehistorica Hipana XXII. Madrid. C.S.I.C.

CABrera, V. y Bischoff, J. (1989): "Accelerator 14C dates for Early Upper Paleolithic (Basal Aurignacian) at El Castillo Cave (Spain)", Journal of Archeological Science, .16, págs. 577-584.

CLARK, J.G.D. (1972): «Palaeolithic butchery practices», Ucko, Thrigham y Dimberley, Man, Settlement and Urbanism, págs. 149-156.

DART, R. (1957): The osteodontoqueratic culture of Australopithecus prometeus, Transvaal Museum Memoir 10. Pretoria. 
LARTET, E. (1860): "On the coexistance of man with certain extinct quadrupeds, proved by fossil bones, from varios Pleistocene deposits, bearing incisions made by sharp instruments" (reed.: Heizer, Man's discovery of his past).

Lyman, R. L. (1987): "Archaeofaunas and Butchery Studies: A taphonomic Perspective", Advances in archaeological method and theory, vol. 10. New York, págs. 249-337.

Pumarejo, P. G., e.p.: «Restos óseos con incisiones en la Cueva de A Valiña (Lugo). Notas para su estudio". Soto Berreiro, M. J. y Llana, C., Cova da Valiña (Castroverde, Lugo) Un yacimiento del Paleolítico Superior Inicial en Galicia (Campañas de 1987 y 1988). Xunta de Galicia.

Pumarejo, P. G. y Bernaldo de Quiros, F. (1990): "Huellas humanas en huesos. Análisis de sus implicaciones económicas (1) . Revista de Arqueologia. n." 108, págs. 16-24.

- (1990): "Huellas humanas en huesos. Análisis de sus implicaciones económicas (II)". Revista de Arqueologia, n. ${ }^{\circ} 109$, pags. 14-24.

REIXACH CASALS, J. (1986): "Huellas antrópicas. Metodología, diferenciación y problemática». Revista de Arqueologia, n. ${ }^{\circ}$ 60, págs. 6-14. 